\title{
Change in hospitalization rates following transcatheter left atrial appendage occlusion
}

Moghniuddin Mohammed ${ }^{1}$, Sharma Kattel², Irfan Ahsan ${ }^{3}$, Abdul J. Samdani $^{4}$, Devesh Rai ${ }^{4}$, Swati Chand ${ }^{4}$, Dhrubajyoti Bandyopadhyay ${ }^{5}$, Sagar Ranka ${ }^{1}$, Amit Noheria ${ }^{1}$, Sanjaya K. Gupta ${ }^{6}$

\author{
${ }^{1}$ Department of Cardiovascular Medicine, The University of Kansas Health System, \\ Kansas City, KS, USA \\ ${ }^{2}$ Section of Cardiovascular Medicine, Department of Internal Medicine, Yale School \\ of Medicine, New Haven, CT, USA \\ ${ }^{3}$ Department of Internal Medicine, Geisinger Medical Center, Danville, PA, USA \\ ${ }^{4}$ Department of Cardiology, Sands-Constellation Heart Institute, Rochester Regional \\ Health, Rochester, NY, USA \\ ${ }^{5}$ Department of Cardiology, Westchester Medical Center, NY, USA \\ ${ }^{6}$ Department of Cardiology, Saint Luke's Mid America Heart Institute, Kansas City, MO, \\ USA
}

Submitted: 3 August 2021

Accepted: 30 August 2021

Arch Med Sci Atheroscler Dis 2021; 6: e191-e195

DOI: https://doi.org/10.5114/amsad.2021.111405

Copyright (c) 2021 Termedia \& Banach

\begin{abstract}
Introduction: Left atrial appendage occlusion (LAAO) is recommended in patients with non-valvular atrial fibrillation (AF) who have contraindications to or are intolerant of long-term oral anticoagulants (OAC), but its impact on hospitalization rates has not been well described. The objective of our study is to describe the incidence of all-cause, bleeding-related, and thrombosis-related hospitalizations before and after LAAO.

Material and methods: We used the Nationwide Readmission Database to include patients aged $\geq 18$ years with a diagnosis of AF who underwent transcatheter LAAO during the months of February-November in each year between 2016 and 2018. Patients who died during the index procedure or had missing length of hospital stay or mortality information were excluded. Results: A total of 27,633 patients were included (median age: 77 years, $41 \%$ female) with an average pre- and post-LAAO monitoring period of 6.5 and 5.5 months respectively. Of these, 10,808 (39.1\%) patients had one or more admissions prior to the procedure compared to 7,196 (26.0\%) after the procedure. There was a $26 \%$ reduction in incidence of all-cause admissions (rate ratio $(\mathrm{RR})=0.74,95 \%$ confidence interval $(\mathrm{Cl}): 0.71-0.76 ; p<0.001)$, $49 \%$ reduction in bleeding-related admissions ( $R R=0.51,95 \% \mathrm{Cl}: 0.48-0.55$; $p<0.001$ ), and $71 \%$ reduction in thrombosis-related readmissions ( $R R=$ $0.29,95 \% \mathrm{Cl}: 0.26-0.33 ; p<0.001)$ after LAAO.

Conclusions: In a contemporary, nationally representative dataset, we found that LAAO is associated with a significant decrease in all-cause, bleeding-related, and thrombosis-related admissions. These findings lend support to the current use of transcatheter LAAO in clinical practice for patients with contraindications to OAC and/or at high risk of bleeding.
\end{abstract}

Key words: left atrial appendage occlusion, stroke, bleeding, outcomes, hospitalizations, atrial fibrillation.

\author{
Corresponding author: \\ Devesh Rai \\ Department of Cardiology \\ Sands-Constellation Heart \\ Institute \\ Rochester Regional Health \\ Rochester, NY, USA \\ E-mail: deveshraiMD@gmail. \\ com
}




\section{Introduction}

Atrial fibrillation (AF) is the most common sustained arrhythmia and is associated with a 5-fold increased risk of stroke [1]. Oral anticoagulants (OAC) are the standard of care for ischemic stroke prevention and the availability of direct OAC (DOAC) has broadened the therapeutic options. However, this benefit comes with an increased risk of bleeding, and the trials evaluating DOAC excluded patients with high risk of bleeding or who had suffered a recent bleeding event $[2,3]$ Studies have shown that approximately 1 in $8 \mathrm{AF}$ patients have an accepted contraindication to OAC and approximately $50 \%$ of patients with AF at moderate to high risk of stroke are not on OAC $[4,5]$, making them vulnerable to both ischemic and hemorrhagic events in the absence of an alternative treatment [2].

Left atrial appendage occlusion (LAAO) has been shown to be non-inferior to warfarin and DOAC for preventing thromboembolic events $[6,7]$ and currently has a class $\mathrm{Ilb}$ recommendation for use in non-valvular AF patients with contraindications to long-term OAC $[8,9]$. To date, no study has examined the pre- and post-LAAO hospitalization rates. To address this knowledge gap, we analyzed the incidence of all-cause, bleeding-related, and thrombosis-related hospitalizations in patients undergoing LAAO.

\section{Material and methods}

This is a retrospective cohort study using the Nationwide Readmission Database (NRD) from January 2016 to December 2018. Briefly, the NRD is a nationally representative dataset that includes hospitalization data from 28 states representing approximately $59 \%$ of all U.S. hospitalizations for each years [10]. Because NRD data are not linked between years, we included adult patients (age $\geq 18$ ) discharged alive after transcatheter LAAO during the months of February to November to ensure at least 30 days of pre-and post-procedural monitoring in any given year. NRD is a unique database which does not report the exact date of admission to protect patient identity, but marks the patient's admission on an arbitrary time scale, such that time interval between admissions can be computed. Using the months of admission, reported in the database, we could compute the pre-procedural and postprocedural time periods. As exact dates were unknown and median LOS was 1 day, we assumed that each person's procedure was performed exactly in the middle of a month, such that there were 0.5 months on either side of the day of the procedure. For example, if a person underwent the procedure in the $5^{\text {th }}$ month (May), then we assumed that the patient had 4.5 months of pre-procedural and 7.5 months of post-procedural monitoring. For patients who died on readmission, actual days to death were counted, as NRD facilitates such calculation, and then divided by 30 to obtain the post-procedure time in months.

All analyses were performed taking into account the complex sampling design as recommended by the Healthcare Cost and Utilization Project (HCUP). Continuous variables were presented as median (IQR) and categorical variables were presented as percentages and frequencies. All admission counts and person-time were derived from weighted data. Hierarchical negative binomial regression models were unweighted as per the recommendations of the HCUP [11] and included patients and hospitals as random effects. The results were similar with and without hospital being included as a random effect and the final parsimonious model without hospital was used to report the incidence rate ratios (RR). Person-months was included as an offset variable to account for variable periprocedural time contribution by individual patients. The International Classification of Diseases, 10th edition codes (ICD-10) used are shown in Supplementary Table SI.

We also performed a sensitivity analysis by creating a blanking period of 2 months post-procedurally, which is generally the time frame over which patients are on OAC after transcatheter LAAO to prevent device-related thrombosis.

\section{Statistical analysis}

All analyses were performed using $R$ version 3.6.1. The 'Survey' package was used to account for the complex sampling design and the 'Ime4' package was used for hierarchical regression [12, 13]. Two-sided $p<0.05$ was considered statistically significant.

\section{Results}

The final cohort consisted of 27,633 patients with a median age of 77 years $(71.0,82.0)$ and 11,467 (41\%) being female (Figure 1). Cardiovascular co-morbidities were common with a median

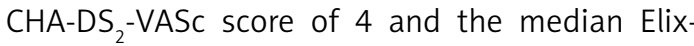
hauser comorbidity score was $4[3,5]$. The median length of stay (LOS) and cost of index hospitalization were 1 day and \$25,006 (18,929-30,827), respectively (Table I). The average pre-procedural monitoring period was 6.5 months (0.54 years) and the average post-procedural monitoring period was 5.5 months ( 0.46 years). The total person-time was 14,699 person-years pre-procedurally and 12,844 person-years post-procedurally.

Overall, there were 17,572 admissions pre-procedurally, with the most frequent causes being gastrointestinal tract bleeding (GIB) (16.9\%), car- 


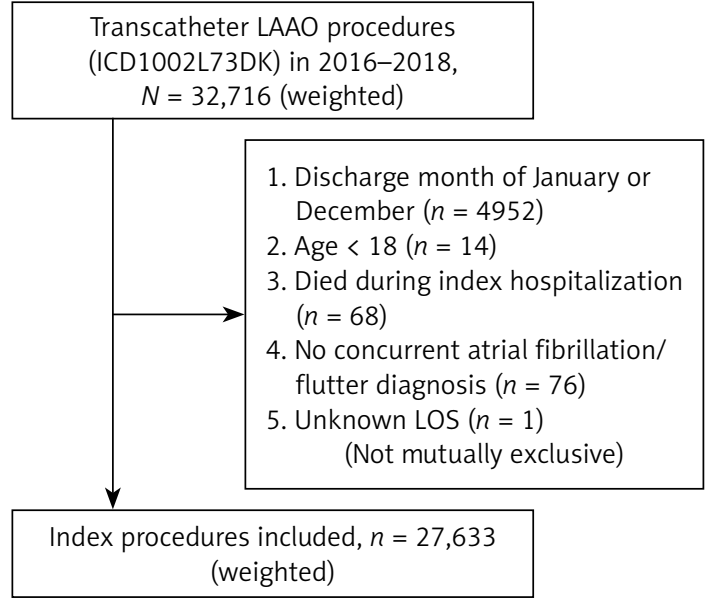

Figure 1. Patient flow diagram with inclusion and exclusion criteria for index admission

diac arrhythmias (11.7\%), congestive heart failure (CHF) (9.5\%) and ischemic stroke/transient ischemic attack (TIA) (7.5\%). There was a total of 11,264 admissions post-procedurally, with the most common cause being CHF (14.5\%) followed by GIB (12.7\%). Ischemic stroke/TIA accounted for 315 (2.8\%) admissions after the procedure. Intracranial hemorrhage $(\mathrm{ICH})$ related admissions accounted for $4 \%$ of total admissions pre-procedurally and $1.4 \%$ post-procedurally.

Of the 27,633 patients, 10,808 (39.1\%) patients had one or more admissions prior to the procedure compared to 7,196 (26.0\%) patients after the procedure (Figure 2 ). There was a $26 \%$ reduction in all-cause incidence of post-procedure admissions ( $\mathrm{RR}=0.74,95 \% \mathrm{Cl}: 0.71-0.76 ; p \leq 0.001)$. The incidence of bleeding-related admissions was lower by $49 \%(\mathrm{RR}=0.51,95 \% \mathrm{Cl}: 0.48-0.55 ; p<$ $0.001)$ and that of thrombosis-related admissions was lower by $71 \%(R R=0.29,95 \% \mathrm{Cl}: 0.26-0.33$; $p<0.001$ ) (Figure 3).

After accounting for the blanking period, we found an even greater reduction in the incidence of bleeding-related admissions ( $R R=0.27,95 \% \mathrm{Cl}$ : $0.25-0.3 ; p<0.001$ ) (Figure 3).

\section{Discussion}

The major findings of our study are:

1. GIB is the most common cause of pre-LAAO hospitalization.

2. After LAAO, there was a significant reduction in the incidence of bleeding and thrombosis-related admissions.

3. After accounting for the blanking period, there was an even greater reduction in the incidence of bleeding-related admissions.

Both DOAC and LAAO are associated with reduction in hemorrhagic stroke in comparison to warfarin [14]. In contrast, the DOAC do not or only marginally reduce other major bleeding re-
Table I. Baseline characteristics of the study population

\begin{tabular}{|c|c|}
\hline Parameter & Results \\
\hline Total population & 27633 \\
\hline Age, median [IQR] & $77.0[71.0,82.0]$ \\
\hline Female & $11467(41)$ \\
\hline \multicolumn{2}{|l|}{ Median household income: } \\
\hline $0-25^{\text {th }}$ percentile & $5876(22)$ \\
\hline $26^{\text {th }}-50^{\text {th }}$ percentile & $6928(25)$ \\
\hline $51^{\text {st }}-75^{\text {th }}$ percentile & $7713(28)$ \\
\hline $76^{\text {th }}-100^{\text {th }}$ percentile & $6786(25)$ \\
\hline \multicolumn{2}{|l|}{ Expected primary payer: } \\
\hline Medicare & $24719(90)$ \\
\hline Medicaid & $264(1)$ \\
\hline Private insurance & $2182(8)$ \\
\hline Congestive heart failure & $10625(38)$ \\
\hline Valvular heart disease & $6058(22)$ \\
\hline Peripheral vascular disease & 4709 (17) \\
\hline Hypertension & $23835(86)$ \\
\hline Diabetes & $9747(35)$ \\
\hline Chronic pulmonary disease & $5870(21)$ \\
\hline Chronic kidney disease & $6375(23)$ \\
\hline Liver disease & $750(3)$ \\
\hline Peptic ulcer disease & $204(1)$ \\
\hline Obesity & $4506(16)$ \\
\hline $\mathrm{CHA} \mathrm{DS}_{2}$-VASc, median [IQR] & $4.0[3.0,5.0]$ \\
\hline $\mathrm{CHADS}_{2}$, median [IQR] & $2.0[2.0,3.0]$ \\
\hline Elixhauser score, median [IQR] & $4.0[3.0,5.0]$ \\
\hline \multicolumn{2}{|l|}{ Weighted Charlson index: } \\
\hline 0 & $6113(22)$ \\
\hline $1-2$ & $12101(44)$ \\
\hline $3-4$ & $5887(21)$ \\
\hline$\geq 5$ & $3533(13)$ \\
\hline LOS, median [IQR] & $1.0[1.0,1.0]$ \\
\hline Cost, in US dollars, median [IQR] & $\begin{array}{c}25006 \\
{[18929,30827]}\end{array}$ \\
\hline
\end{tabular}

lated to the GI tract, bladder, and skin [14]. GIB is seen in $5-15 \%$ of patients receiving long-term OAC and is the most commonly occurring major bleeding complication [3]. Moreover, in the ARISTOTLE trial, the history of GIB was associated with an increased risk of subsequent major GIB on anticoagulation [15]. In our cohort, at least $15 \%$ of patients experienced 1 or more bleeding-related hospitalization before LAAO, with GIB being the most common cause.

The incidence of ICH-related hospitalizations was also reduced after $\mathrm{LAAO}$, as observed in the trials and registries evaluating Watchman [7]. 
A

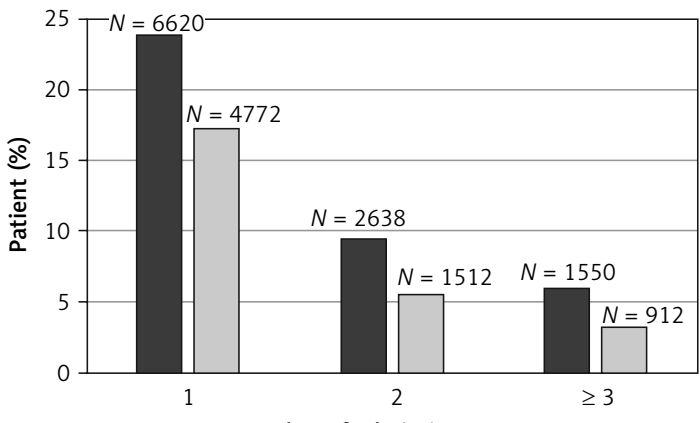

Number of admissions

C

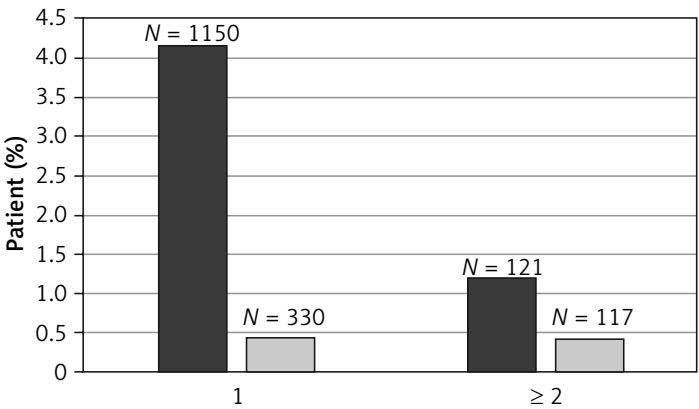

Number of admissions

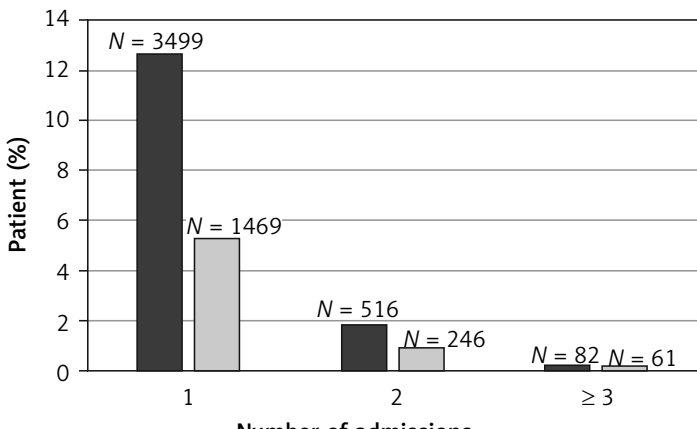

Number of admissions

D

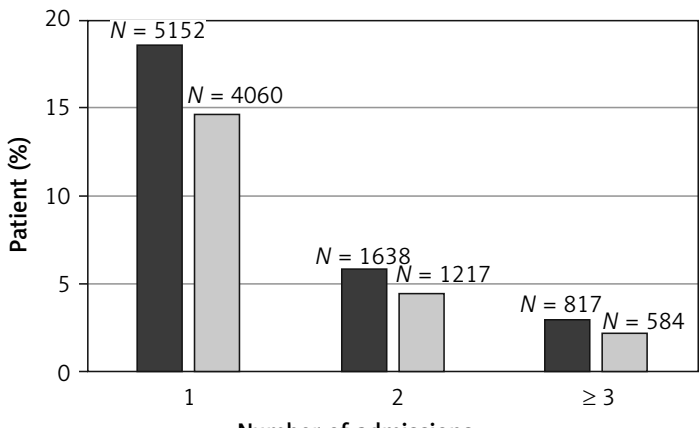

Number of admissions

$\square$ Pre-LAAO $\square$ Post-LAAO

Figure 2. Comparison of pre- and post-procedure admission counts. Figure shows the percent of patients on the Y-axis stratified by admission count on the X-axis: A - all cause admissions, $\mathbf{B}$ - bleeding related admissions, $\mathbf{C}$ - thrombosis related admissions, $\mathbf{D}$ - no bleeding and non-thrombosis related admissions

Readmission reason Pre-procedure Post-procedure

Rate ratio $(95 \% \mathrm{Cl}) \quad P$-value

No. of admissions

(incidence per 100 person-years)

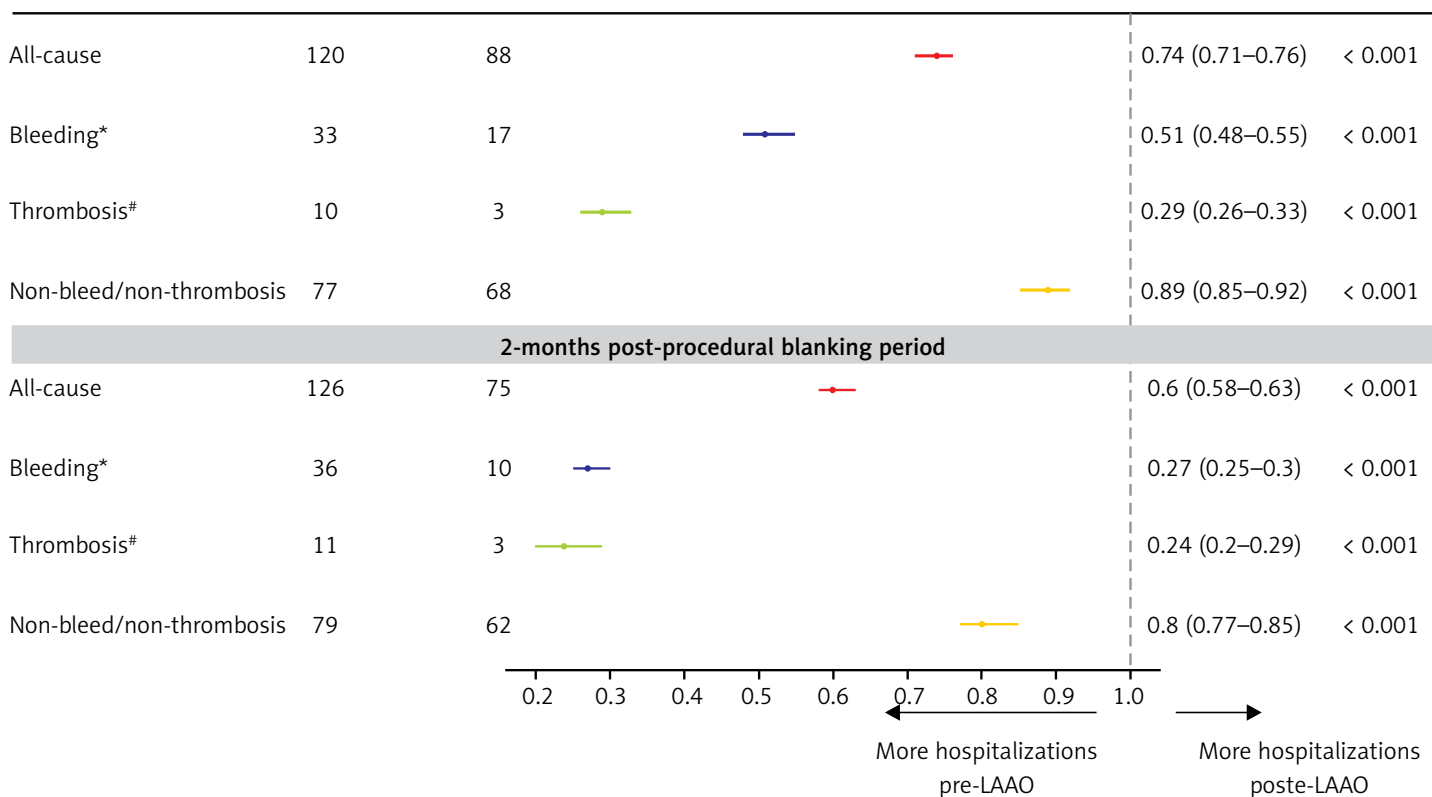

Figure 3. Incidence rate ratio (RR) stratified by cause of admission for the entire monitoring duration and with 2-month post-procedural blanking period. Figure shows the RR with associated $95 \% \mathrm{Cl}$ and $p$-values

${ }^{*}$ Bleeding-related admissions include gastrointestinal bleeding, intracranial, post-procedural and bleeding at other sites (e.g. hemarthrosis). "Thrombosis-related admissions include ischemic stroke, intracardiac thrombi, intestinal, renal, other arterial ischemia and device-related thrombosis. 
Additionally, we found a greater reduction in the incidence of bleeding-related hospitalization after accounting for the blanking period of 2 months. Several trials are evaluating post-procedural antithrombotic regimens along the spectrum of OAC candidacy, including the ASAP-TOO trial, in which patients will not receive any OAC after the procedure [14]. This might potentially lead to a further decrease in post-LAAO bleeding events.

Similar to previous studies, we found a significant decrease in the incidence of ischemic stroke, with a concomitant reduction in bleeding events. In addition, we found that non-bleeding-related and non-thrombosis-related hospitalizations also decreased, which reassures that the benefit of LAAO in terms of bleeding-/thrombosis-related hospitalizations is not counterbalanced by complication-related hospitalizations.

NRD is an administrative database and is subject to possible miscoding for cause of hospitalization. Our study cannot account for patients who died out of hospital, and thus may underestimate the totality of the morbidity and mortality associated with transcatheter LAAO. All analyses are limited to one calendar year, and long-term outcomes could not be assessed, but our pre- and post-procedure windows of 6.5 and 5.5 months were long enough to capture substantial rates of hospitalization in this high-risk patient population. Finally, all patients in our study underwent transcatheter LAAO, and we therefore cannot assess the incidence of hospitalizations in medically managed patients in the absence of this procedure.

In conclusion, using a contemporary, nationally representative real-world dataset, we found that transcatheter LAAO is associated with a significant decrease in all-cause, bleeding-related and thrombosis-related hospitalizations. These findings lend support to the current use of transcatheter LAAO in clinical practice for patients with contraindications to OAC and/or at high risk of bleeding. Future studies are needed to evaluate the cost-effectiveness of LAAO.

\section{Conflict of interest}

The authors declare no conflict of interest.

\section{References}

1. Virani SS, Alonso A, Benjamin EJ, et al.; American Heart Association Council on E, Prevention Statistics C, Stroke Statistics S. Heart Disease and Stroke Statistics-2020 Update: A Report From the American Heart Association. Circulation 2020; 141: e139-596.

2. Redfors B, Gray WA, Lee RJ, Ellenbogen KA, Bonafede M, Ben-Yehuda O. Patients with atrial fibrillation who are not on anticoagulant treatment due to increased bleeding risk are common and have a high risk of stroke. JACC Clin Electrophysiol 2017; 3: 1369-76.
3. Witt DM. What to do after the bleed: resuming anticoagulation after major bleeding. Hematology Am Soc Hematol Educ Program 2016; 2016: 620-4.

4. Hsu JC, Maddox TM, Kennedy KF, et al. Oral anticoagulant therapy prescription in patients with atrial fibrillation across the spectrum of stroke risk: insights from the NCDR PINNACLE Registry. JAMA Cardiol 2016; 1: 55-62.

5. Xian Y, O'Brien EC, Liang L, et al. Association of preceding antithrombotic treatment with acute ischemic stroke severity and in-hospital outcomes among patients with atrial fibrillation. JAMA 2017; 317: 1057-67.

6. Osmancik P, Herman D, Neuzil P, et al.; Investigators P-T. Left atrial appendage closure versus direct oral anticoagulants in high-risk patients with atrial fibrillation. J Am Coll Cardiol 2020; 75: 3122-35.

7. Holmes DR Jr, Doshi SK, Kar S, et al. Left atrial appendage closure as an alternative to warfarin for stroke prevention in atrial fibrillation: a patient-level meta-analysis. J Am Coll Cardiol 2015; 65: 2614-23.

8. January CT, Wann LS, Calkins H, et al. 2019 AHA/ACC/ HRS Focused Update of the 2014 AHA/ACC/HRS Guideline for the Management of Patients With Atrial Fibrillation: A Report of the American College of Cardiology/ American Heart Association Task Force on Clinical Practice Guidelines and the Heart Rhythm Society in Collaboration With the Society of Thoracic Surgeons. Circulation 2019; 140: e125-51.

9. Kirchhof P, Benussi S, Kotecha D, et al.; Group ESCSD. 2016 ESC Guidelines for the management of atrial fibrillation developed in collaboration with EACTS. Eur Heart J 2016; 37: 2893-962.

10. NRD Overview. Healthcare Cost and Utilization Project (HCUP). December 2019. Agency for Healthcare Research and Quality, Rockville, MD., December 2019.

11. Houchens RCB, Steiner C. Hierarchical Modeling using HCUP Data HCUP Methods Series Report \# 2007-01 Online: U.S. Agency for Healthcare Research and Quality, January 10, 2007.

12. Lumley T. Analysis of complex survey samples. J Statistical Software 2004; 9: 19.

13. Bates D, Mächler M, Bolker B, Walker S. Fitting linear mixed-effects models Usinglme4. J Statistical Software 2015; 67.

14. Holmes DR, Reddy VY, Buchbinder M, et al. The Assessment of the watchman device in patients unsuitable for oral anticoagulation (ASAP-TOO) trial. Am Heart J 2017; 189: 68-74.

15. Garcia DA, Fisher DA, Mulder H, et al. Gastrointestinal bleeding in patients with atrial fibrillation treated with Apixaban or warfarin: Insights from the Apixaban for Reduction in Stroke and Other Thromboembolic Events in Atrial Fibrillation (ARISTOTLE) trial. Am Heart J 2020; 221: 1-8. 